\title{
To which extent does immigration affect inequality?
}

\author{
Yonatan Berman \\ School of Physics and Astronomy, Tel-Aviv University, Tel-Aviv, Israel \\ Department of Computer Science, University College London, London, UK \\ E-mail: yonatanb@post.tau.ac.il \\ and Tomaso Aste \\ Department of Computer Science, University College London, London, UK \\ E-mail: t.aste@ucl.ac.uk
}

\begin{abstract}
The current surge in income and wealth inequality in most western countries, along with the continuous immigration to those countries demand a quantitative analysis of the effect immigration has on economic inequality. This paper presents a quantitative analysis framework providing a way to calculate this effect. It shows that in most cases, the effect of immigration on wealth and income inequality is limited, mainly due to the relative small scale of immigration waves. For a large scale flow of immigrants, such as the immigration to the US, the UK and Australia in the past few decades, we estimate that $10 \% \div 15 \%$ of the wealth and income inequality increase can be attributed to immigration. The results demonstrate that immigration could possibly decrease inequality substantially, if the characteristics of the immigrants resemble the characteristics of the destination middle class population in terms of wealth or income. We empirically found that the simple linear relation $\Delta S=0.18 \rho$ roughly describes the increase in the wealth share of the top $10 \%$ due to immigration of a fraction $\rho$ of the population.
\end{abstract}

Keywords: econophysics, immigration, wealth inequality, income inequality

Submitted to: New J. Phys.

\section{Introduction}

The recent surge in wealth and income inequalities is one of the most disturbing social and economic issues of our time. The rapid increase in inequality, illustrated for the US in figure 1, has generated much effort to understand the origin and possible control of this trend and a wide variety of factors have been proposed as effectors determining the dynamics of inequality. These factors range across the changes in social structure and culture $[1,2,3]$, the democratization of education [4], the rise of the financial 


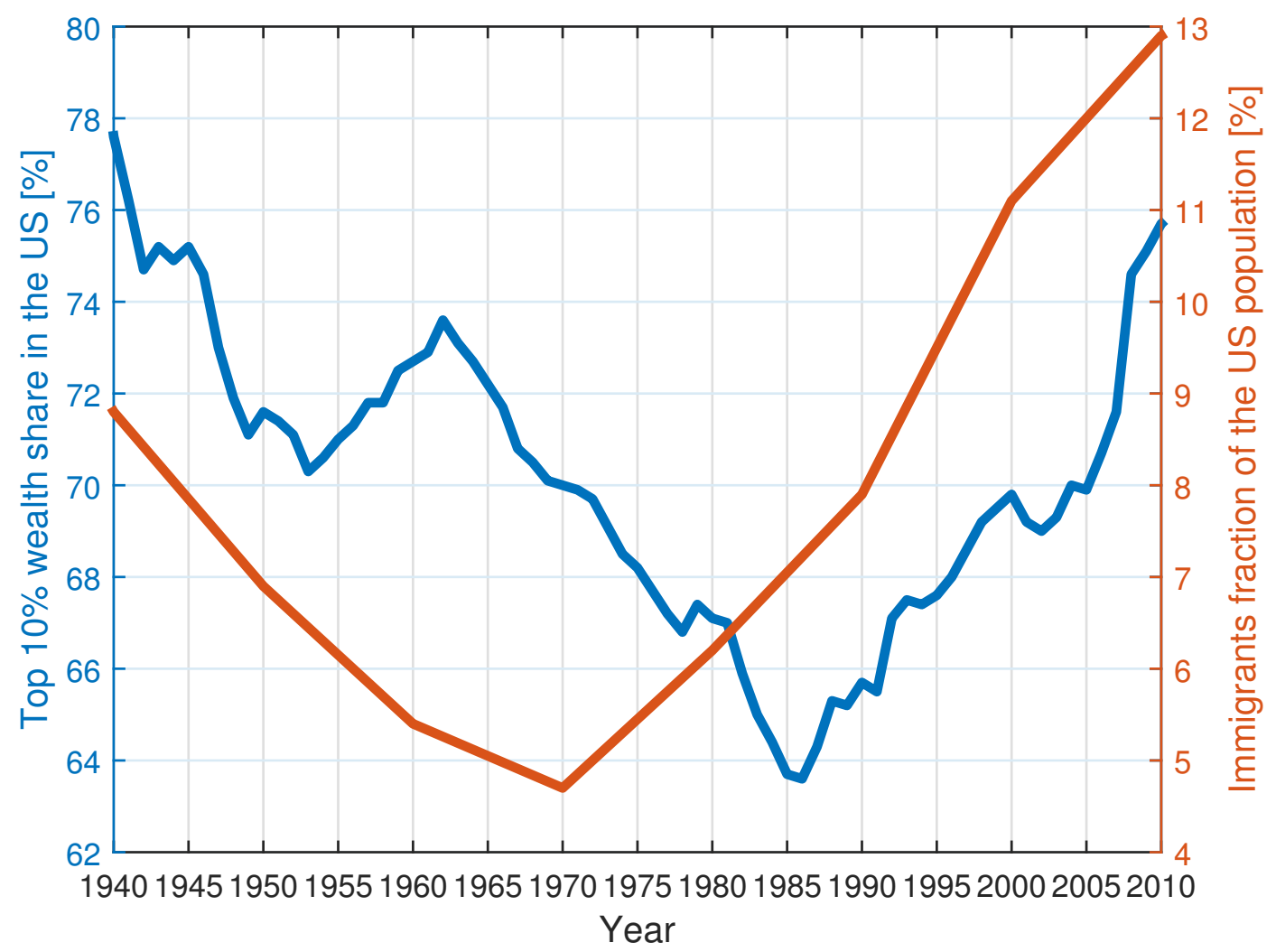

Figure 1. US wealth inequality and immigrant population in the US 1940-2010. The share of wealth owned by the richest $10 \%$ (blue) and the immigrants fraction of the US population (red). The inequality data are taken from [8]. The immigration data are based on the US census data and taken from [13].

sector [5], exploitation rents and the character of institutions [2, 6, 7], tax policy $[2,3,8]$, bequests $[9,10]$, and the dynamics of personal savings [11, 12].

Immigration is considered as one of the factors contributing to wealth and income inequalities in developed countries. The immigration rates to many developed countries have generally increased in the past few decades and immigrants constitute a growing fraction of the population, as illustrated in figure 1. As a result, the outcomes of immigration are becoming increasingly important to their economies and labor markets.

Borjas [14] has exposed a significant effect of immigration on the wages of native workers in the US (see also $[15,16,17,18]$ ), specifically on the wages of natives with no high school diploma. Card's analyses [19, 20] provide a similar methodology, partially supporting these results by showing that immigrants are found to be perfect substitutes for native workers with up to a high school equivalent level of education but imperfect substitutes in the case of college equivalent or higher education level. Ottaviano and Peri [21] have recently argued that immigration had a small effect on the wages of native workers with no high school degree and also a small positive effect on average native wages and a substantial negative effect on wages of previous immigrants in the long run. Rienzo and Vargas-Silva [22] have found that income inequality among immigrants 
in the US and the UK is higher than among natives. The general conclusions of all of these studies were that although immigration has an effect on wage inequality and on the distribution of income, it only accounts for a small fraction of the increase in inequality. It was recently reported by Dustmann et al [23] that immigration "depresses wages below the 20th percentile of the wage distribution but leads to slight wage increases in the upper part of the wage distribution". Card [20] has also suggested that immigration accounted for $5 \%$ of the wage inequality increase between 1980 and 2000 in the US. HIbbs et al [24] have recently presented a much higher figure of 24\%, while other studies estimated contributions of about $10 \%$ to inequality due to immigration in recent years $[25]$.

Much less attention was given to the effect immigration has on wealth inequality. Ben-Gad [26] provided a theoretical framework demonstrating that the role of immigration in the redistribution of income from labor to capital is small, supporting some of conclusions drawn by Borjas [15]. Hao [27] has argued that immigration plays a little part in the increase of wealth inequality in the past few decades in the US. Hao's analysis is based on a similar methodology to the previous studies of Borjas and Card on income inequality, discussing the part education and skill play in wealth accumulation processes. This study suggests that "lower-skill recent immigrants actually help to maintain the share of the low educated, thereby contributing to only a tiny percentage of the rising inequality".

Multiple studies $[14,16,22,28,29,30]$ describe a large variety of immigration types, with different characteristics in terms of skill, education and wealth. Specifically, Borjas [17], Card [19] and Rienzo and Vargas-Silva [22] have shown that the increased immigration to developed countries in the past few decades is usually characterized by a varied background in terms of skills, education and socio-economic level, and is generally much poorer than the population in the destination country. The effect immigration waves with different characteristics have on inequality is naturally dependent on those characteristics. Nevertheless, a quantitative estimation of the change in inequality originated directly from immigration can be theoretically derived, under certain assumptions on the immigrants characteristics.

In this paper we present a simple framework for quantitatively assessing the direct effect of immigration on inequality. We do so by calculating the effect of "injecting" a smaller population into a larger population on inequality measures. We consider different scenarios reflecting various immigration characteristics. Such a calculation can be used to evaluate the future effect of possible immigration scenarios, and equally important, to quantitatively assess what part of the current increase in wealth and income inequality in many countries can be attributed to the net immigration into these countries. The presented framework significantly differs from methodologies used in the described recent studies. In our case, we do not aim to demonstrate a statistically significant effect by measuring wages and wealth values. Instead, we calculate directly the change in inequality based on a few general assumptions on the immigrants characteristics and on the wealth and income distribution of the destination country. We then use our 
theoretical findings for comparison with empirical data of inequality and immigration in order to validate the calculations and the method in general. This way we can provide meaningful estimations of the effect in discussion.

The rest of the paper is organized as follows. section 2 introduces the analysis framework and its underlying assumptions. In section 3, we present the theoretical results for the different immigration scenarios and under various sets of assumptions. In section 4 we then validate and compare the calculations to empirical data on immigration and inequality in different countries. section 5 concludes the paper and discusses the results and their implications.

\section{Methodology}

The starting point of our theoretical approach is two populations - $X$ and $Y$, which represent two countries, or any two entities characterized by distributions $P_{X}$ and $P_{Y}$, respectively. These distributions can be either wealth or income distributions. We will analyse the direct effect on the inequality measures of $Y$, following the migration of $n=\rho N_{Y}$ individuals from $X$ to $Y . N_{X}$ and $N_{Y}$ are the population sizes of $X$ and $Y$, respectively. Let us denote by $\tilde{X}$ (with distribution $P_{\tilde{X}}$ ) the migrating population and by $\tilde{Y}$ the $Y$ population following the migration, as illustrated in figure 2 .

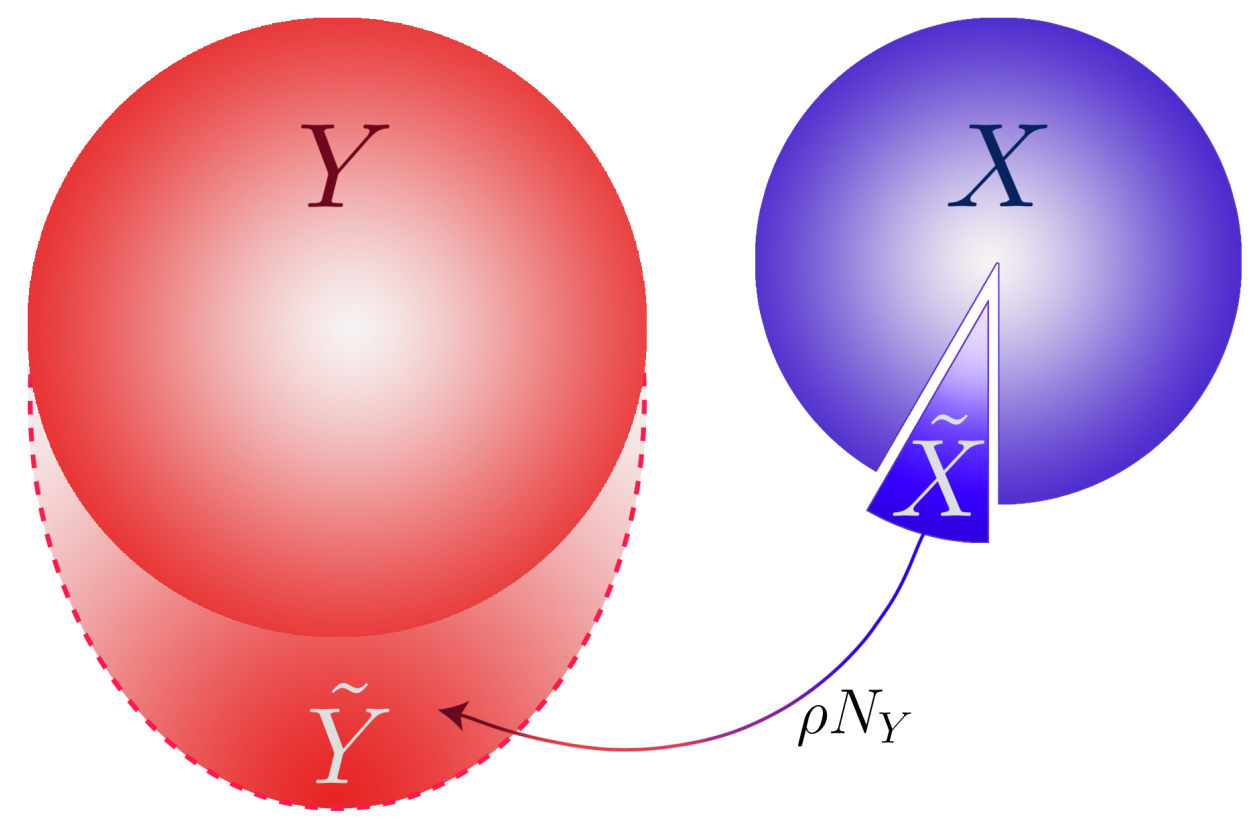

Figure 2. Illustration of the analysis framework. Two populations are analysed $X$ and $Y$. We denote by $\tilde{X}$ the migrating population from $X$, consisting of $\rho N_{Y}$ individuals, where $N_{Y}$ is the original size of the $Y$ population. We denote by $\tilde{Y}$ the $Y$ population following the migration, which is the union of $Y$ and $\tilde{X}$.

The probability distribution of $\tilde{Y}$ is

$$
P_{\tilde{Y}}(x)=\frac{N_{Y}}{N_{Y}+n} P_{Y}(x)+\frac{n}{N_{Y}+n} P_{\tilde{x}}(x)=\frac{1}{1+\rho}\left[P_{Y}(x)+\rho P_{\tilde{x}}(x)\right],
$$


and the cumulative distribution function is therefore

$$
F_{\tilde{Y}}(x) \equiv \int_{0}^{x} P_{\tilde{Y}}\left(x^{\prime}\right) d x^{\prime}=\frac{1}{1+\rho} \int_{0}^{x} P_{Y}\left(x^{\prime}\right)+\rho P_{\tilde{x}}\left(x^{\prime}\right) d x^{\prime} .
$$

To quantify inequality we will use the share of wealth/income owned by a top wealth/income quantile $q, S(q)$ or specifically the top decile $(q=0.1)$. Cowell [31] discusses a wide variety of measures, concluding that none is universally superior to the others. Therefore, we shall restrict our discussion to $S(q)$, which is one of the most extensively used measures (see, for example, $[3,8,12,32]$ ).

The share of wealth/income owned by the top quantile $q$ is

$$
S_{\tilde{Y}}(q)=\left(1-\frac{\int_{0}^{1-q} x\left(F_{\tilde{Y}}\right) d F_{\tilde{Y}}}{\int_{0}^{1} x\left(F_{\tilde{Y}}\right) d F_{\tilde{Y}}}\right)=\frac{\int_{1-q}^{1} x\left(F_{\tilde{Y}}\right) d F_{\tilde{Y}}}{\mu_{F_{\tilde{Y}}}},
$$

where $x\left(F_{\tilde{Y}}\right)$ is the inverse function of $F_{\tilde{Y}}(x)$ and $\mu_{F_{\tilde{Y}}}=\int_{0}^{1} x\left(F_{\tilde{Y}}\right) d F_{\tilde{Y}}$ is the average wealth/income in the population $\tilde{Y}$.

A closed form expression for $S_{\tilde{Y}}(q)$ in the general case cannot be derived. In order to obtain closed form expressions for the inequality measures it is necessary to make certain limiting assumptions on the distributions of $X$ and $Y$. An analytic derivation for the case in which $X$ and $Y$ follow a Pareto distribution is given in Appendix A. However, for practical purposes, numerical simulations can be performed in order to calculate $S(q)$ and compare it to the corresponding share of wealth of the original population of $Y$.

We will consider 3 different immigration scenarios reflecting different types and profiles of immigration waves:

- Random immigration - The $n$ migrating individuals are randomly chosen from $X$

- Rich immigration - The $n$ richest individuals of $X$ are migrating to $Y$

- Poor immigration - The $n$ poorest individuals of $X$ are migrating to $Y$

The poor and rich immigration scenarios, as defined above, are extreme scenarios. Indeed, in practice, rich immigration waves are not characterized by the migration of the whole rich elite. Therefore, the analysis will provide a bound for the effect of immigration in this case. Poor immigration waves are also not characterized by the total migration of lower castes. However, this is less important quantitatively, since in this scenario, the contribution of the migrating population to the total wealth or income is very small.

Each of the populations $X$ and $Y$ is initially described by the following properties:

- Population size - $N$

- Inequality - $S(0.1)$ - defined as the share of wealth or income owned by the top $10 \%$

- Mean wealth/income - $\mu$ (the inequality measures depend on the distribution shape and are invariant under multiplication by constant. Therefore, we will only consider the ratio $\eta$ between the mean wealth/income of population $X$ to this of $Y$ ) 
- Distribution shape

We assume that both $P_{X}$ and $P_{Y}$ follow a log-normal distribution, which is considered a representative distribution of wealth and income $[12,33,34,35,36]$. For a log-normal distribution the cumulative distribution function is $F(x)=\frac{1}{2}+\frac{1}{2} \operatorname{erf}\left(\frac{\ln x-\mu}{\sqrt{2} \sigma}\right)$ and its inverse is $x(F)=e^{\mu+\sqrt{2} \sigma \cdot \operatorname{erf}^{-1}(2 F-1)}$. Therefore, there is a one-to-one correspondence between the parameter $\sigma$ and $S(q)$ through $S(q)=\frac{\int_{1-q}^{1} e^{\sqrt{2} \sigma \cdot \operatorname{erf}^{-1}(2 F-1)} d F}{\int_{0}^{1} e^{\sqrt{2} \sigma \cdot \operatorname{erf}^{-1}(2 F-1)} d F}$ (see equation (3)). Using this relation we can obtain the value of $\sigma$ for the distributions of $X$ and $Y$ corresponding to the desired initial inequality of these populations.

For the poor immigration scenario, it is possible to derive a simpler expression for $\Delta S=S_{\tilde{Y}}(0.1)-S_{Y}(0.1)$. Indeed, the contribution of the poor immigrants to the total wealth of the $Y$ population is negligible so we assume that it remains unchanged. Therefore, the share of wealth owned by the top $10 \%$ in $\tilde{Y}$ will slightly increase, as more individuals $\left(\frac{n}{10}=\rho \frac{N_{Y}}{10}\right)$ are now a part of the top decile, due to the change in the population size. As a result, the value of $\Delta S$ will simply be the share of wealth of the $\rho \frac{N_{Y}}{10}$ richest individuals in $Y$ that are not a part of the top decile of $Y$, which yields to:

$$
\Delta S \approx \frac{\int_{0.9-\frac{\rho}{10}}^{0.9} x\left(F_{Y}\right) d F_{Y}}{\mu_{Y}}
$$

This result implies that for any given distribution of $Y$, the effect of poor immigration is essentially dependent on the value of $\rho$ only. For small enough and realistic values of $\rho$ (namely $<0.1$ ) the dependence of $\Delta S$ on $\rho$ is approximately linear with a slope depending on the initial distribution of $Y$. This is originated in the approximate power law characterizing the tail of wealth and income distributions, for which the integral in equation (4) is proportional to $\rho^{1-\chi}$, for a small $\chi$, which depends on the power law exponent. When the initial inequality of $Y$ is very high, the contribution of the $\rho \frac{N_{Y}}{10}$ individuals added to the top decile is relatively low and vice versa. Therefore, we would expect the linear dependence of $\Delta S$ on $\rho$ to be steeper as the initial inequality of $Y$ decreases.

A first order expansion of equation (4) for the case of Pareto distribution with tail index $\alpha$ has the explicit form (see Appendix B):

$$
\Delta S \approx\left(1-\frac{1}{\alpha}\right) 0.1^{1-\frac{1}{\alpha}} \cdot \rho
$$

We note that the Pareto distribution is a better representation of the higher levels of wealth and income than the log-normal distribution and therefore applicable for the scenario of poor immigration (the log-normal distribution is a better representation of the distribution overall, while the Pareto distribution is only a better representation of the high levels of income and wealth). Several studies have established that the characteristic values for $\alpha$ in the case of wealth and income distributions are $1.5-3[34,35,36,37,38]$, which yield to slopes of $0.14-0.16$ in equation (5). 
We will now proceed to make a comprehensive and systematic analysis using numerical calculations of equation (3).

\section{Results}

In order to systematically analyse the immigration effect on inequality we will consider $Y$ as a rich population characterized by high inequality $\left(S_{Y}(0.1)=75 \%\right)$ and consider $X$ to be a poorer population for two cases: one in which $X$ is characterized by high inequality (similar to $Y$ ) and another in which inequality is significantly lower $\left(S_{X}(0.1)=60 \%\right)$. For all the different cases and scenarios we will vary the scale of the immigration $(\rho)$ and the relative wealth of $X$ with respect to $Y, \eta=\mu_{X} / \mu_{Y}$.

In addition, since the initial distributions are randomly created and, in the random immigration scenario, the migrating individuals are randomly selected, the calculation results will be slightly different in each run for the same parameters. Therefore, 500 different realizations were considered for every case and we calculated the average change in $S(q)$, so that the results are statistically robust. The results of this analysis are presented in figure 3, where we can observe that for the random and rich immigration scenarios both negative and positive effects on inequality are possible. In both scenarios the reduction of inequality is possible by significantly increasing the share of wealth owned by the "middle class", i.e. by the population which is close to the median wealth. Such a reduction is achieved when two conditions are fulfilled:

- A significant amount of wealth enters the economy, either when the migrating population scale is large enough, or when the wealth of $X$ is comparable to that of $Y$

- The migrating population is not rich enough to be a part of the rich elite of $Y$

The results for the poor immigration scenario confirm the derivation of $\Delta S$ for this scenario in equation (4), demonstrating that $\Delta S$ is linearly dependent on the scale of the immigration $(\rho)$ and is essentially independent of the distribution of $X$. In order to quantify the dependence of $\Delta S$ on $\rho$, we calculated it for different values of $S_{Y}(0.1)$. These results are presented in figure 4 and show that for every additional $1 \%$ of poor immigrants, the share of wealth owned by the top $10 \%$ will increase by $0.15 \div 0.2$ percentage points. For most relevant cases, the slope is approximately 0.18 , so the change in the share of wealth owned by the top $10 \%$ roughly follows $\Delta S=0.18 \rho(\Delta S$ is measured in percentage points and $\rho$ in percent). We note that these slopes are close to the approximate dependence on $\rho$ derived for $\Delta S$ given a Pareto distribution of $Y$ (see equation (5)).

The results enable a quantitative estimation of the effect of immigration on inequality, showing that for most cases, the effect of the different scenarios is limited within \pm 1 percentage points. A larger effect can be achieved for the rich immigration scenario if the immigration scale is very large (close or larger than 5\%) or if the average wealth of $X$ is close to the average wealth of $Y$. Illustrative scenarios for such cases can 

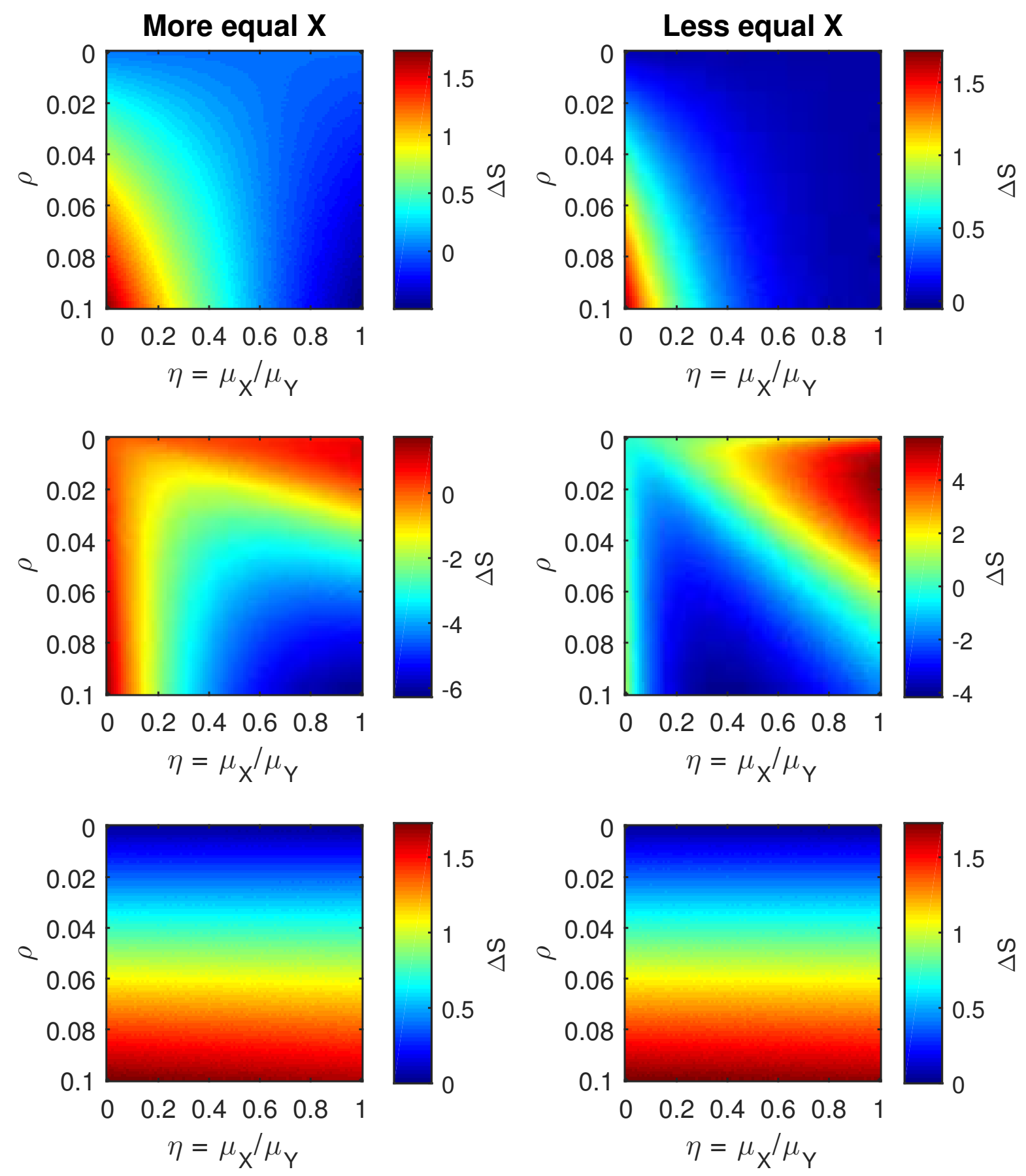

Figure 3. The change in the wealth share owned by the top $10 \%$ in population $Y$ following the migration from population $X$ to population $Y$. The calculations were done for the two cases - low inequality $X\left(S_{X}(0.1)=0.6\right)$ (left) and high inequality $X$ $\left(S_{X}(0.1)=0.75\right)$ (right) for the 3 immigration scenarios - random (top), rich (middle) and poor (bottom). For each of these cases the dependence of $\Delta S$ is on $\eta$ - the ratio between the average wealth of $X$ and $Y$ and on $\rho$ - the fraction of the migrating population. $\Delta S$ is measured in percentage points.

be the hypothetical immigration of the richest 10\% elite of Spain to France, which will significantly reduce the wealth inequality in France, or the immigration of the richest 1\% 

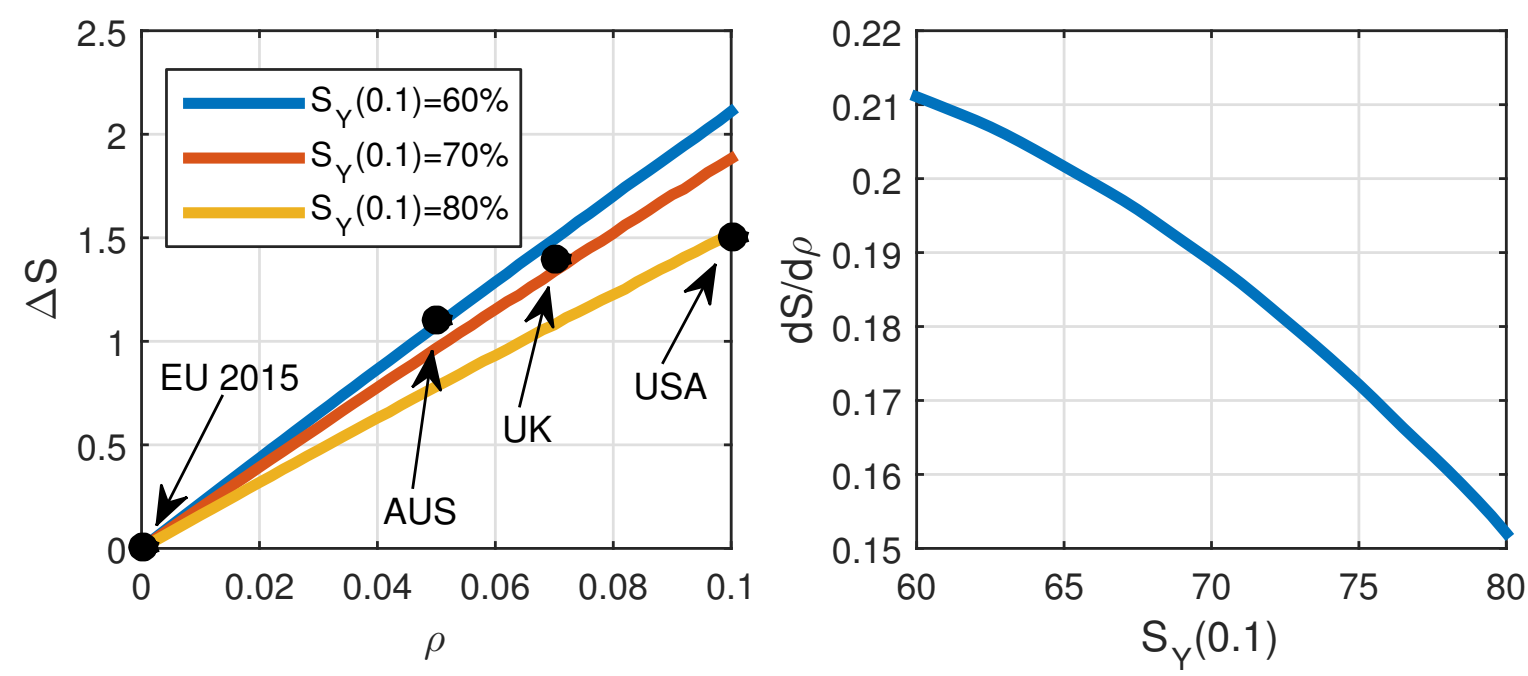

Figure 4. The dependence of $\Delta S$ on $\rho$. (Left) The change in inequality for the poor immigration scenario as a function of the immigration scale (controlled by the parameter $\rho$ ). The black circles refer to a comparison with empirical evidence for several cases investigated in section 4; (Right) Based on the linear dependence of $\Delta S$ on $\rho$ in the poor immigration scenario, the slope of this relationship is presented for different values of the initial $S_{Y}(0.1)$.

elite of Denmark to Switzerland which will significantly increase the wealth inequality in Switzerland. However, in practice, rich immigration waves tend to be very small in scale, so that their effect on inequality is likely to be relatively small as well.

\section{Comparison with empirical data}

In order to support the above findings, we compared the results with empirical data. We present below a few examples for which historical data are available. These examples, illustrated in figure 4, demonstrate that the linear relation $\Delta S=0.18 \rho$ is generally applicable as a first order approximation to estimate the effect of immigration on inequality.

- The immigration to the US during 1986-2012 consisted of approximately $26 \cdot 10^{6}$ immigrants, originating mainly from Mexico $\left(5.5 \cdot 10^{6}\right)$, China, India and the Philippines [39, 40], which are approximately 10\% of the US population in 1986. Saez and Zucman [8] report that over this time period, the share of wealth owned by the top $10 \%$ in the US increased by approximately 14 percentage points to $77 \%$. Due to the varied origin countries of the immigrants and their varied socio-economic background, this immigration can be treated as random. However, Guillermina et al report in the New Immigration Survey [41] that the wealth owned by immigrants upon their arrival to the US, specifically from Mexico, China, India and the Philippines, is very low when compared to the average wealth in the US. As a result, in terms of our analysis, it can be considered as a poor immigration scenario. Based 
on equation (3) and the results reported in figure 3, this immigration wave can explain an increase of approximately 1.5 percentage points from the 14 percentage points increase in inequality, or a relative part of $11 \%$ of the increase. In the case of income inequality, Piketty and Saez [32] show that the top decile share of income had increased during 1986-2012 by approximately 12 percentage points. Using the same analysis it is possible to attribute $15 \%$ of this increase to immigration. This is in agreement with various estimations conducted by economists using different models and methodologies providing results ranging from $5 \%$ to $24 \%$ of the increase in income inequality [25].

- In the UK, approximately $4 \cdot 10^{6}$ immigrants were added to the working age population between 1990 to 2014 [42]. This immigration period corresponds roughly to a random immigration wave of $7 \%$ of the population. Rienzo and VargasSilva [22] show that these immigrants are originated from various countries and backgrounds, but are considered to be mostly low skilled and poor. This is also supported by the data reported in the Labor Force Survey [43]. Piketty [3] argues that during this period, the share of wealth owned by the top $10 \%$ in the UK increased by approximately 10 percentage points. Using equation (3), based on our analysis framework and considering the poor immigration scenario, immigration can be attributed to an increase of approximately 1.4 percentage points of the total 10 percentage points increase, or simply $14 \%$ of the increase. This analysis is consistent with the empirical findings in [22]. Restricting the analysis to a shorter period of time, it was found that in the past few years, an average of approximately $2 \cdot 10^{5}$ net immigrants came to the UK every year ( $0.3 \%$ of the total UK population). Applying the analysis framework and equation (3), this translates to an increase of 0.06 percentage points in the top $10 \%$ share of income, which explains $12 \%$ of the annual increase in income inequality in the past few years in the UK, which is approximately 0.5 percentage points per year, as reported by Belfield et al [44].

- Australia is another country attracting a large number of immigrants. The Australian Bureau of Statistics reports that during 1995-2005 Australia received approximately $10^{6}$ immigrants, about $5 \%$ of its population [45]. Stilwell [46] argues that during the same time period, the share of wealth owned by the top $10 \%$ increased from approximately $42.5 \%$ to $52 \%$. Based on these data, and applying the analysis framework for the poor immigration scenario, immigration can be attributed to an increase of approximately 1.1 percentage points of the total 9.5 percentage points increase, or $12 \%$ of the increase. This result is consistent with the effect found for the other large scale immigration waves analysed in the US and the UK.

- The massive immigration of refugees and asylum seekers from Syria to Europe, mainly during the second half of 2015 is largely composed of poor and middle class Syrians. The UN [47] reported that of $4.6 \cdot 10^{6}$ registered refugees, approximately $8 \cdot 10^{5}$ are in Europe, as of November 2015. Applying the analysis framework 
for the poor immigration scenario, it is possible to estimate that for every $10^{5}$ immigrants to Europe, the share of wealth and income owned by the top $10 \%$ will increase by approximately 0.004 percentage points. Hence, this immigration wave has contributed to a small increase of approximately 0.03 percentage points in the share of wealth owned by the top $10 \%$ in Europe, so far.

\section{Discussion}

We presented a systematic quantitative analysis framework to estimate the direct effect of immigration on inequality. The application of this framework for the analysis of the large scale immigration to the US, the UK and Australia in the past few decades provides an explanation for $10 \% \div 15 \%$ of the increase in income and wealth inequality measures. This estimation is consistent with most of the empirical studies done for the US, as reported by Borjas [16] and Nowrasteh [25]. In the case of poor-profiled immigrants, which represents a large fraction of the immigration waves in the world, we estimate an increase of $0.15 \div 0.2$ percentage points in the top $10 \%$ share of wealth and income for every $1 \%$ of the population added by immigration, or roughly $\Delta S=0.18 \rho$ (see section 4 and figure 4). This simple linear relationship was demonstrated as an applicable first order approximation to the increase in wealth and income inequality due to the immigration of a fraction $\rho$ of the population.

The main conclusion of the analysis is that in most realistic cases, in which the immigrating population is poor compared to destination population, the direct effect of immigration on inequality is relatively small. This conclusion is consistent with specific analyses done for the income inequality in the US [14, 19] and supports more recent studies advocating for easier immigration policies and open borders in many areas in the world [23, 48, 49].

The rich immigration scenario creates the most dramatic effect on inequality of the scenarios considered, and provides a bound of approximately \pm 5 percentage points change in the share of wealth owned by the top $10 \%$, even in the case of a very large scale immigration wave (10\% of the destination country). We also note that in some cases, large scale immigration of rich individuals from a poorer population to a richer population can significantly decrease inequality. As a consequence, international immigration programs intended to attract rich individuals and families, such as the Canada Business Immigration Program and Immigrant Investor Venture Capital Pilot Program or the US EB-5 Immigrant Investor Program, may lead to a reduction in wealth inequality [50, 51, 52]. However, in practice, their small scale compared to the overall population, makes this effect essentially negligible.

Our results are based on simple statistical findings and do not consider interactions between the immigrant population and the destination population. This is, of course, a limitation of our study, but also makes our results general and independent from any immigration dynamics. As such, our results can be considered as a null interaction benchmark. A more detailed analysis can consider positive or negative deviations from 
this benchmark and identify contributions of immigrants to the destination population wealth and income distributions. Furthermore, our analysis provides only an estimation of the direct effect of immigration on economic inequality. In the long run, inequality measures are governed mainly by different factors related to the destination country economic situation and policies $[2,3,7]$. Such policies could enhance or reduce the direct effect immigration has on inequality. We note, however, that wealth accumulation processes are relatively slow [12,33], supporting the robustness of our results and their validity. An empirical evidence for that is the analysis of annual immigration data for the UK, found to be consistent with the results for a 24-years period (see section 4).

In addition, for our analysis we used the top $10 \%$ share of wealth and income as measures of inequality. Similar results are obtained for the Gini coefficient, as presented in Appendix C. Other measures of inequality can be used, and might dramatically affect the results. For example, using the ratio of the share of income or wealth owned by the top $10 \%$ and the bottom $10 \%$, would result in a very different estimation in the case of the poor immigration scenario. As the source population gets poorer, the increase in inequality due to immigration would be higher. Hence, in practice, care should be taken when measuring inequality and applying the analysis results for different scenarios. The framework presented here can be easily generalized to any inequality measure derived from the income or wealth distributions. Therefore, it provides a simple but powerful analysis tool to estimate the initial effect of immigration on inequality under various scenarios and assumptions.

\section{Acknowledgments}

We thank Michael Ben-Gad for his elaborate feedback on the manuscript, and for Cinzia Rienzo and Delmiro Fernandez-Reyes for fruitful discussions. 


\section{Appendix A. Derivation of the quintile wealth share for Pareto distribution}

Let us first assume that $X$ and $Y$ follow a Pareto distribution, which describes well higher levels of income and wealth, but not lower levels:

$$
P_{X}(x)= \begin{cases}0 & x<x_{m} \\ \frac{\alpha x_{m}^{\alpha}}{x^{\alpha+1}} & x \geq x_{m}\end{cases}
$$

while $P_{Y}(x)$ follows a similar distribution with different values of $x_{m}$ and $\alpha$. We will assume $x_{m}$ is identical, and only assume two different $\alpha$ values $\alpha_{1}$ and $\alpha_{2}$ corresponding to the distributions of $X$ and $Y$, respectively. In addition, we assume the initial sizes of $X$ and $Y$ may be different $-N_{X}$ and $N_{Y}$ respectively.

We will derive an analytic closed form expression for the rich immigration scenario. A similar derivation can be applied for the poor immigration scenario. In the case in which $X$ and $Y$ follow the exact same distribution, random immigration is, naturally, meaningless in terms of wealth or income inequality.

For rich immigration, we can define $\epsilon$ as the lowest wealth value within the $n$ migrating individuals. $\epsilon$ is a function of the distribution parameters $x_{m}$ and $\alpha_{1}$ and also depends on $N_{X}$ and $n=\rho N_{Y}$. As a result, the new population $\tilde{Y}$, of size $N_{Y}+n$, follows a slightly different distribution:

$$
P_{\tilde{Y}}(x)= \begin{cases}0 & x<x_{m} \\ \frac{\alpha_{2} x_{m}^{\alpha_{2}}}{x^{\alpha_{2}+1}} \frac{N_{Y}}{N_{Y}+n} & x_{m} \leq x<\epsilon . \\ \frac{\alpha_{2} x_{m}^{\alpha_{2}}}{x^{\alpha_{2}+1}} \frac{N_{Y}}{N_{Y}+n}+\frac{\alpha_{1} \epsilon^{\alpha_{1}}}{x^{\alpha_{1}+1}} \frac{n}{N_{Y}+n} & x \geq \epsilon\end{cases}
$$

In order to calculate the inequality measures of $\tilde{Y}$, the cumulative distribution function and its inverse function should be derived. Following equation (A.2) we obtain by integration and some algebra:

$$
F_{\tilde{Y}}(x)=\left\{\begin{array}{ll}
0 & x<x_{m} \\
\left(1-\frac{x_{m}^{\alpha_{2}}}{x^{\alpha_{2}}}\right) \frac{N_{Y}}{N_{Y}+n} & x_{m} \leq x<\epsilon . \\
1-\frac{1}{N_{Y}+n}\left(\frac{n \epsilon^{\alpha_{1}}}{x^{\alpha_{1}}}+\frac{N_{Y} x_{m}^{\alpha_{2}}}{x^{\alpha_{2}}}\right) & x \geq \epsilon
\end{array} .\right.
$$

From equation (A.3) it is now possible to derive the inverse function of the cumulative distribution function. For the general case of $\alpha_{1} \neq \alpha_{2}$ one cannot obtain a 
To which extent does immigration affect inequality?

closed form expression for $x\left(F_{\tilde{Y}}\right)$. Assuming $\alpha_{1}=\alpha_{2}=\alpha$ we obtain:

$$
F_{\tilde{Y}}(x)=\left\{\begin{array}{ll}
0 & x<x_{m} \\
\left(1-\frac{x_{m}^{\alpha}}{x^{\alpha}}\right) \frac{N_{Y}}{N_{Y}+n} & x_{m} \leq x<\epsilon, \\
1-\frac{1}{N_{Y}+n}\left(\frac{n \epsilon^{\alpha}+N_{Y} x_{m}^{\alpha}}{x^{\alpha}}\right) & x \geq \epsilon
\end{array},\right.
$$

and therefore

$$
x\left(F_{\tilde{Y}}\right)=\left\{\begin{array}{ll}
\frac{x_{m}}{\left(1-F_{\tilde{Y}} \tilde{N}\right)^{\frac{1}{\alpha}}} & 0 \leq F_{\tilde{Y}}<F_{\epsilon} \\
\tilde{x}{\frac{1}{1-F_{\tilde{Y}}}}^{\frac{1}{\alpha}} & F_{\epsilon} \leq F_{\tilde{Y}}<1
\end{array},\right.
$$

while $\tilde{N}=\frac{N_{Y}+n}{N_{Y}}=1+\rho, \tilde{x}=\left[\frac{n \epsilon^{\alpha}+N_{Y} x_{m}^{\alpha}}{\left(N_{Y}+n\right)}\right]^{\frac{1}{\alpha}}=\left[\frac{\rho \epsilon^{\alpha}+x_{m}^{\alpha}}{(1+\rho)}\right]^{\frac{1}{\alpha}}$ and $F_{\epsilon}=\left(1-\frac{x_{m}^{\alpha}}{\epsilon^{\alpha}}\right) \frac{1}{\tilde{N}}$.

The share of wealth owned by the top quantile $q$ can be simply calculated by taking $S(q)=\left(1-\frac{\int_{0}^{1-q} x\left(F_{\tilde{Y}}\right) d F}{\int_{0}^{1} x\left(F_{\tilde{Y}}\right) d F}\right)$. We will calculate several useful sub-integrals of the integrals used for the calculation of $S(q)$.

$$
\begin{aligned}
\int_{0}^{F_{\epsilon}} x(F) d F & =\int_{0}^{F_{\epsilon}} \frac{x_{m}}{(1-F \tilde{N})^{\frac{1}{\alpha}}} d F= \\
& =\frac{\alpha x_{m}}{\tilde{N}(\alpha-1)}\left[1-\left(1-F_{\epsilon} \tilde{N}\right)^{1-\frac{1}{\alpha}}\right]= \\
& =\frac{\alpha x_{m}}{\tilde{N}(\alpha-1)}\left[1-\left(\frac{x_{m}^{\alpha}}{\epsilon^{\alpha}}\right)^{1-\frac{1}{\alpha}}\right]
\end{aligned}
$$

If $F_{\epsilon}>1-q$ we get:

$$
\begin{aligned}
\int_{0}^{1-q} x(F) d F & =\int_{0}^{1-q} \frac{x_{m}}{(1-F \tilde{N})^{\frac{1}{\alpha}}} d F= \\
& =\frac{\alpha x_{m}}{\tilde{N}(\alpha-1)}\left[1-(1-\tilde{N}+\tilde{N} q)^{1-\frac{1}{\alpha}}\right]
\end{aligned}
$$

In addition we should calculate the following integrals:

$$
\int_{F_{\epsilon}}^{1} x(F) d F=\int_{F_{\epsilon}}^{1} \tilde{x}{\frac{1}{1-F_{\tilde{Y}}}}^{\frac{1}{\alpha}} d F=\frac{\tilde{x} \alpha}{\alpha-1}\left(1-F_{\epsilon}\right)^{1-\frac{1}{\alpha}},
$$

and (given that $F_{\epsilon}<1-q$ )

$$
\int_{F_{\epsilon}}^{1-q} x(F) d F=\int_{F_{\epsilon}}^{1-q} \tilde{x} \frac{1}{1-F_{\tilde{Y}}} \frac{\frac{1}{\alpha}}{\alpha-1} d F=\frac{\tilde{x} \alpha}{\alpha-1}\left[\left(1-F_{\epsilon}\right)^{1-\frac{1}{\alpha}}-q^{1-\frac{1}{\alpha}}\right]
$$


To which extent does immigration affect inequality?

There are two cases - if $F_{\epsilon}>1-q$ we obtain:

$$
\begin{aligned}
S(q) & =1-\frac{\int_{0}^{1-q} x(F) d F}{\int_{0}^{F_{\epsilon}} x(F) d F+\int_{F_{\epsilon}}^{1} x(F) d F}= \\
& =1-\frac{\frac{x_{m}}{\tilde{N}}\left[1-(1-\tilde{N}+\tilde{N} q)^{1-\frac{1}{\alpha}}\right]}{\frac{x_{m}}{\tilde{N}}\left[1-\left(\frac{x_{m}^{\alpha}}{\epsilon^{\alpha}}\right)^{1-\frac{1}{\alpha}}\right]+\tilde{x}\left(1-F_{\epsilon}\right)^{1-\frac{1}{\alpha}}},
\end{aligned}
$$

and if $F_{\epsilon}<1-q$ we obtain:

$$
\begin{aligned}
S(q) & =1-\frac{\int_{0}^{F_{\epsilon}} x(F) d F+\int_{F_{\epsilon}}^{1-q} x(F) d F}{\int_{0}^{F_{\epsilon}} x(F) d F+\int_{F_{\epsilon}}^{1} x(F) d F}= \\
& =1-\frac{\frac{x_{m}}{\tilde{N}}\left[1-\left(\frac{x_{m}^{\alpha}}{\epsilon^{\alpha}}\right)^{1-\frac{1}{\alpha}}\right]+\tilde{x}\left[\left(1-F_{\epsilon}\right)^{1-\frac{1}{\alpha}}-q^{1-\frac{1}{\alpha}}\right]}{\frac{x_{m}}{\tilde{N}}\left[1-\left(\frac{x_{m}^{\alpha}}{\epsilon^{\alpha}}\right)^{1-\frac{1}{\alpha}}\right]+\tilde{x}\left(1-F_{\epsilon}\right)^{1-\frac{1}{\alpha}}}= \\
& =\frac{\tilde{x} q^{1-\frac{1}{\alpha}}}{\frac{x_{m}}{\tilde{N}}\left[1-\left(\frac{x_{m}^{\alpha}}{\epsilon^{\alpha}}\right)^{1-\frac{1}{\alpha}}\right]+\tilde{x}\left(1-F_{\epsilon}\right)^{1-\frac{1}{\alpha}} .}
\end{aligned}
$$

For a standard Pareto distribution $S(q)=q^{1-\frac{1}{\alpha}}$ and in the trivial case in which $\epsilon=x_{m}$ and $n=0$, the result for $\tilde{Y}$ is reduced to the standard case.

Let us consider a numeric example. We let $\alpha=1.2, x_{m}=1, N_{X}=N_{Y}=10^{7}$ and $n=10^{4}$. In that case $\epsilon=316.5, F_{\epsilon}=0.998$ and $\tilde{x}=1.78$ and according to equation (A.11) the share of wealth owned by the richest $10 \%$ is 0.76 . The initial share of wealth owned by the top $10 \%$ in $Y$ was approximately 0.68 . These results were confirmed by a numerical simulation.

We note that the insights that can be deduced from this derivation are limited for several reasons:

- The Pareto distribution is a good description of high levels of wealth but not of low levels of wealth. Hence it cannot be simply applied for poor immigration waves.

- The analytic solution is limited to the same value of $\alpha$ and $x_{m}$, and cannot be used for modeling immigration between completely different economies. The numeric example given may provide insight on the possible impact of the Moscowian wealthiest elite hypothetical immigration to London, but not on the impact of the immigration of Syrian middle class immigration to Germany. 


\section{Appendix B. Derivation of $\Delta S$ for Pareto distribution in the poor immigration scenario}

According to equation (4), the change in the wealth share of the top $10 \%$ under the poor immigration scenario follows

$$
\Delta S \approx \frac{\int_{0.9-\frac{\rho}{10}}^{0.9} x\left(F_{Y}\right) d F_{Y}}{\mu_{Y}},
$$

where $x\left(F_{Y}\right)$ is the inverse function of the cumulative distribution function $F_{Y}(x)$ and $\mu_{Y}$ is the average wealth in the population $Y$.

If $Y$ follows a Pareto distribution with tail index $\alpha$ and scale parameter $x_{m}$, the inverse cumulative distribution function follows:

$$
x\left(F_{Y}\right)=\frac{x_{m}}{\left(1-F_{Y}\right)^{\frac{1}{\alpha}}}
$$

and the average is:

$$
\mu_{Y}=\frac{\alpha x_{m}}{\alpha-1}
$$

It follows that

$$
\begin{aligned}
\Delta S & \approx \frac{\int_{0.9-\frac{\rho}{10}}^{0.9} x\left(F_{Y}\right) d F}{\mu_{Y}}= \\
& =\frac{\int_{0.9-\frac{\rho}{10}}^{0.9} \frac{x_{m}}{\left(1-F_{Y}\right)^{\frac{1}{\alpha}}} d F_{Y}}{\frac{\alpha x_{m}}{\alpha-1}}=(0.1+0.1 \rho)^{1-\frac{1}{\alpha}}-0.1^{1-\frac{1}{\alpha}} .
\end{aligned}
$$

Following the first order Taylor expansion of $(0.1+0.1 \rho)^{1-\frac{1}{\alpha}}-0.1^{1-\frac{1}{\alpha}}$ around $\rho=0$ we obtain

$$
\Delta S \approx\left(1-\frac{1}{\alpha}\right) 0.1^{1-\frac{1}{\alpha}} \cdot \rho,
$$

which is equation (5).

\section{Appendix C. The analysis results for the Gini coefficient}

The exact same procedure done for producing the results presented in figure 3 was repeated this time calculating the change in the Gini coefficient of $Y$ following the different immigration scenarios. Considering $G_{Y}$ as the Gini coefficient of $Y$ and $G_{\tilde{Y}}$ as the Gini coefficient of $\tilde{Y}$ we simply calculate and present their difference $\Delta G=G_{\tilde{Y}}-G_{Y}$. We considered the same scenarios and parameters as done for figure 3. These results, presented in figure $\mathrm{C} 1$ demonstrate that all the conclusions made for the share of wealth owned by the top $10 \%$ are applicable for the Gini coefficient as well. 

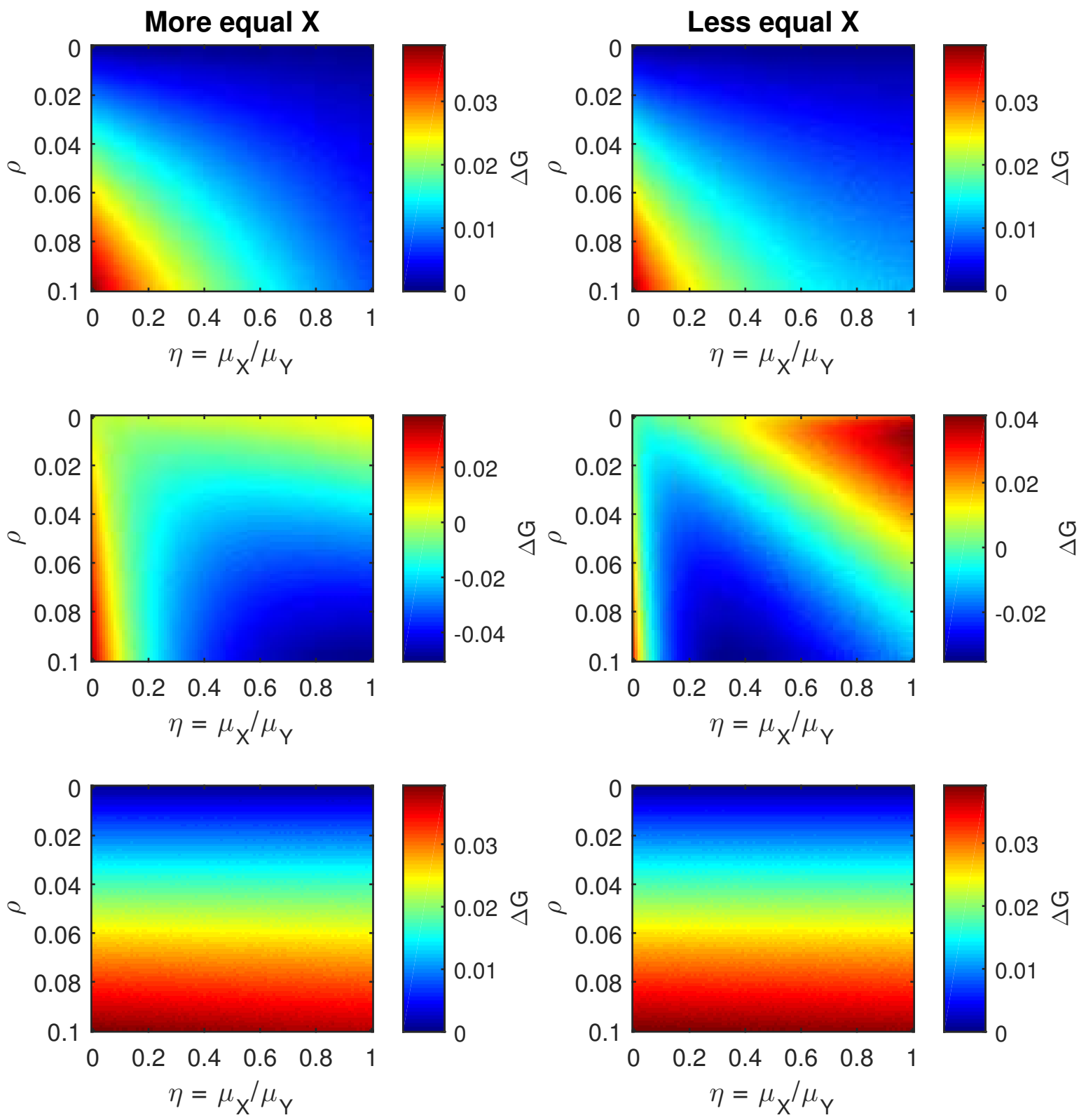

Figure C1. The change in the Gini coefficient in population $Y$ following the migration from population $X$ to population $Y$. The calculations were done for the two cases - low inequality $X\left(G_{X}=0.65\right)$ (left) and high inequality $X\left(G_{X}=0.775\right)$ (right) for the 3 immigration scenarios - random (top), rich (middle) and poor (bottom). For each of these cases the dependence of $\Delta G$ is on $\eta$ - the ratio between the average wealth of $X$ and $Y$ and on $\rho$ - the fraction of the migrating population. 
To which extent does immigration affect inequality?

\section{References}

[1] Keister L A 2000 Wealth in America: Trends in wealth inequality (Cambridge University Press)

[2] Reich R B 2012 Beyond outrage: What has gone wrong with our economy and our democracy, and how to fix it (Vintage)

[3] Piketty T 2014 Cambridge: Harvard Uni

[4] Stiglitz J E 1973 The Annals of the American Academy of Political and Social Science 409 135-145

[5] Stiglitz J 2012 The price of inequality (Penguin UK)

[6] Acemoglu D, Robinson J A and Woren D 2012 Why nations fail: the origins of power, prosperity and poverty vol 4 (SciELO Chile)

[7] Stiglitz J E 2015 National Tax Journal 68 425-448

[8] Saez E and Zucman G 2014 Wealth inequality in the united states since 1913: Evidence from capitalized income tax data Tech. rep. National Bureau of Economic Research

[9] Atkinson A B 1971 Oxford Economic Papers 239-254

[10] De Nardi M 2004 The Review of Economic Studies 71 743-768

[11] Cagetti M and De Nardi M 2008 Macroeconomic Dynamics 12 285-313

[12] Berman Y, Shapira Y and Ben-Jacob E 2015 PloS one 10 e0130181

[13] 2015 Number of immigrants and immigrants as percentage of the u.s. population, 1850 to 2013 Tech. rep. Migration Policy Institute URL http://www.migrationpolicy.org/programs/data-hub/charts/immigrant-population-over-time?width=10008

[14] Borjas G J 2003 The Quarterly Journal of Economics 118 1335-1374 URL http://qje.oxfordjournals.org/content/118/4/1335.abstract

[15] Borjas G J 2001 Heaven's door: Immigration policy and the American economy (Princeton University Press)

[16] Borjas G J and Katz L F 2007 The evolution of the mexican-born workforce in the united states Mexican immigration to the United States (University of Chicago Press) pp 13-56

[17] Borjas G 2013 Center for Immigration Studies, Washington, DC

[18] Borjas G J 2013 IZA Journal of Migration 2 1-25

[19] Card D 2001 Journal of Labor Economics 19 22-64 ISSN 0734306X, 15375307 URL http://www.jstor.org/stable/10.1086/209979

[20] Card D 2009 Immigration and inequality Tech. rep. National Bureau of Economic Research

[21] Ottaviano G I P and Peri G 2012 Journal of the European Economic Association 10 152-197

[22] Rienzo C and Vargas-Silva C 2012 Migration Observatory Briefing, COMPAS, University of Oxford, Oxford

[23] Dustmann C, Frattini T and Preston I P 2013 The Review of Economic Studies 80 145-173

[24] Hibbs B, Hong G et al. 2015 Economics Bulletin 35 650-656

[25] Nowrasteh A 2015 Immigration and economic inequality Cato Institute article URL http://www . cato.org/blog/immigration-economic-inequality

[26] Ben-Gad M 2004 Journal of Economic Dynamics and Control 28 1825-1845

[27] Hao L 2003 New York: Russel Sage Foundation

[28] Daniels R 1990 Coming to America (Harper Collins Publishers)

[29] Portes A 1995 New York: Russell Sage Foundation 29 11-12

[30] Gould E D and Moav O 2014 The Economic Journal

[31] Cowell F 2011 Measuring inequality (Oxford University Press)

[32] Piketty T and Saez E 2013 The Quarterly Journal of Economics 118 1-41

[33] Benhabib J and Bisin A 2007 Work. Pap., New York Univ

[34] Chatterjee A and Chakrabarti B K 2007 European Physical Journal B 60 135-149

[35] Chatterjee A, Sinha S and Chakrabarti B K 2007 Current Science 92 1383-1389

[36] Patriarca M and Chakraborti A 2013 American Journal of Physics 81 618-623

[37] Clementi F and Gallegati M 2005 Physica A: Statistical Mechanics and its Applications $350427-$ 438 
[38] Patriarca M, Chakraborti A and Germano G 2006 Physica A: Statistical Mechanics and its Applications 369 723-736

[39] Baker B and Rytina N 2013 Estimates of the lawful permanent resident population in the united states: January 2013 Tech. rep. US Department of Homeland Security URL http://www.dhs.gov/publication/estimates-lawful-permanent-resident-population-2013

[40] Capps R, Bachmeier J D, Fix M and Van Hook J 2013 Washington, DC: Migration Policy Institute

[41] Guillermina J, Massey D S, Rosenzweig M R and Smith J P 2014 The new immigrant survey 2003 round 2 (nis-2003-2) public release data Tech. rep. The New Immigrant Survey, Princeton University URL http://nis.princeton.edu

[42] Wadsworth J 2015 Immigration and the uk labour market Tech. rep. LSE Centre of Economic Performance URL http://cep.lse.ac.uk/pubs/download/ea019.pdf

[43] 2015 Office for national statistics database URL http://www . ons .gov . uk/ons/publications/index.html

[44] Belfield C, Cribb J, Hood A and Joyce R 2014 Institute for Fiscal Studies, London

[45] 2015 Australian bureau of statistics net overseas migration database URL http://www.abs.gov.au/AUSSTATS/abs@.nsf/DetailsPage/3412.02013-14?OpenDocument

[46] Stilwell F 2014 Australian Options 78 19-22

[47] 2015 Syria regional refugee response URL http://data.unhcr.org/syrianrefugees/regional.php

[48] Bartram D 2010 International Studies Review 12 339-361

[49] Caplan B and Naik V 2014 A radical case for open borders Tech. rep. George Mason University URL http://econfaculty.gmu.edu/bcaplan/caplannaik.pdf

[50] North D 2012 Center for Immigration Studies

[51] Chatterji A, Glaeser E L and Kerr W R 2013 Clusters of entrepreneurship and innovation Tech. rep. National Bureau of Economic Research

[52] Dobrowolsky A 2013 Journal of International Migration and Integration 14 197-218 